\title{
Sarcopenia da caquexia reumatoide: conceituação, mecanismos, consequências clínicas e tratamentos possíveis
}

Oswaldo Melo da Rocha ${ }^{1}$, Andréa de Almeida Peduti Batista ${ }^{2}$, Nailza Maestá3, Roberto Carlos Burini ${ }^{4}$, Iêda Maria Magalhães Laurindo ${ }^{5}$

\begin{abstract}
RESUMO
A caquexia relacionada à artrite reumatoide é conceituada como perda involuntária de massa magra, predominantemente de músculo esquelético, que também ocorre em vísceras e sistema imune, com massa gorda estável ou um pouco elevada e com pequena ou nenhuma perda de peso. A causa é multifatorial, incluindo a produção acentuada de citocinas, principalmente TNF $\alpha$ e Il-1 $\beta$, diminuição da ação periférica da insulina e pouca atividade física. A caquexia se faz presente em doentes com AR ativa ou mesmo inativa. Neste artigo discutem-se aspectos relacionados à patogenia, implicações clínicas e possíveis opções terapêuticas.
\end{abstract}

Palavras-chave: sarcopenia, caquexia, artrite reumatoide, composição corporal.

\section{INTRODUÇÃO}

A musculatura esquelética constitui o maior tecido do corpo, compreendendo a maior massa celular e o maior componente proteico do organismo. ${ }^{1} \mathrm{O}$ tecido muscular, responsável pela autonomia motora do indivíduo, participa não só da homeostase glicêmica e da metabólica, com o suprimento de aminoácidos aos demais tecidos, como também da oxidação das gorduras, fixação do oxigênio e modulação do gasto energético de repouso. ${ }^{1}$

A massa muscular é resultante do equilíbrio entre síntese (anabolismo) e catabolismo (destruição) das suas proteínas, principalmente as miofibrilares. Os fatores catabólicos incluem resistência insulínica e níveis elevados de glicocorticoides, denervação, estresse inflamatório, desuso, restrição calórica, acidose e estresse oxidativo. Além do comprometimento da força, da potência e do equilíbrio, a hipotrofia muscular está associada à resistência insulínica, diabetes mellitus tipo II, hiperadiposidade, menor reparo tecidual e incompetência imunitária. ${ }^{1}$

\section{SARCOPENIA}

A perda de massa muscular associada a prejuízos de função constitui entidade sindrômica denominada sarcopenia. A mais comum é a senil; ${ }^{3-5}$ no entanto, deficiência energética, HIV e doenças inflamatórias crônicas (por exemplo, AR) podem resultar em sarcopenia em indivíduos não idosos. ${ }^{4-7}$

Baumgartner $^{8}$ definiu sarcopenia como diminuição de massa muscular esquelética dois desvios-padrões abaixo da média do grupo-controle constituído por indivíduos jovens (29 anos), saudáveis, pareados para mesma etnia. Baseado nesses critérios, encontrou-se prevalência de $13-24 \%$ na faixa etária de 65 a 70 anos e de mais de $50 \%$ nos indivíduos acima dos 80 anos. A sarcopenia é decorrente da interação de distúrbios da inervação (redução dos motoneurônios acelerada pela grande quantidade de fármacos habitualmente ingerida pelos idosos), diminuição da atividade física, redução de hormônios, aumento dos mediadores inflamatórios e alterações da ingestão proteicocalórica que ocorrem durante o envelhecimento. ${ }^{9-12}$

\footnotetext{
Recebido em 12/09/2008. Aprovado, após revisão, em 02/03/2009. Declaramos a inexistência de conflitos de interesse.

1. Professor Doutor da Disciplina de Reumatologia UNESP

2. Professora Assistente da Disciplina de Reumatologia UNESP

3. Doutora em Nutrição, pós-doutoranda do CeMeNutri UNESP

4. Professor Titular Departamento de Saúde Pública, responsável pelo CeMeNutri UNESP

5. Professora Doutora da Disciplina de Reumatologia FMUSP

Endereço para correspondência: Cristiane Kayser, Rua Botucatu, 740 - $3^{\circ}$ andar, São Paulo, SP, CEP 04023-062. E-mail: criskayser@terra.com.br
} 
É importante frisar que sarcopenia é distinta de caquexia causada por doenças inflamatórias, doenças crônicas avançadas, doenças musculares debilitantes ou desnutrição..$^{12,13,14}$ Nessas situações, ocorrendo a diminuição de massa muscular esquelética (sarcopenia), ela é apenas uma das manifestações da síndrome mais complexa denominada de caquexia. Trata-se, portanto, do componente sarcopênico da caquexia. A síndrome da caquexia é caracterizada pela anorexia, perda de peso, hipoalbuminemia, anemia, alterações da cicatrização de feridas e da imunocompetência. ${ }^{2}$

\section{CAQUEXIA}

Literalmente do grego, caquexia significa condição ruim. Essa palavra habitualmente era relacionada a pacientes em mau estado geral, com doenças consuptivas, estado avançado de desnutrição e depauperação. Atualmente caquexia refere-se à perda de massa celular corpórea por doenças, ${ }^{16}$ sendo acompanhada por perda de massa muscular (componente sarcopênico); deve ser entendida como uma adaptação multidimensional abrangendo grande variedade de alterações, desde mudanças fisiológicas até comportamentais. ${ }^{15}$ Frequentemente pacientes com doenças crônicas ou terminais, como câncer, AIDS, insuficiência cardíaca congestiva, tuberculose, doença pulmonar obstrutiva crônica, fibrose cística, artrite reumatoide (AR), doença de Crohn e outras, apresentam caquexia. ${ }^{15} \mathrm{~A}$ Tabela 1 lista as principais alterações metabólicas encontradas nas caquexias.

A caquexia relacionada à $\mathrm{AR}$ foi descrita pela primeira vez em 1873 por Sir James Paget. ${ }^{17} \mathrm{O}$ termo caquexia reumatoide refere-se à perda de massa corpórea celular e elevado consumo de energia em repouso, que ocorre na $\mathrm{AR}$, e não está necessariamente relacionado ao emagrecimento, uma vez que em muitos pacientes a perda de massa corpórea celular é acompanhada de aumento de massa gorda e o peso continua estável. ${ }^{13,16,19-21}$ Esses casos são conhecidos como caquexia obesa. ${ }^{13,22}$ A perda de massa celular é mais nítida na musculatura esquelética (componente sarcopênico), mas também ocorre nas vísceras e no sistema imune com as consequências ${ }^{22}$ resumidas na Figura 1.

Diagnóstico e classificação do componente sarcopênico da caquexia reumatoide: A presença de caquexia e de seu componente sarcopênico no paciente com $\mathrm{AR}^{23}$ depende da avaliação da composição corporal, que pode ser feita por vários métodos, como ressonância nuclear magnética, tomografia computadorizada, bioimpedância, ultrassonografia, densitometria óssea de corpo total (DXA) e medidas antropométricas. Por exemplo, a massa corpórea celular pode ser medida calculando o potássio corporal total, ${ }^{16,24} \mathrm{e}$ a sua redução é um dos indicadores de caquexia. No momento o método mais utilizado é a densitometria, que permite a avaliação da composição corporal, massa óssea, massa magra e massa adiposa total. ${ }^{9}$ Caso não se disponha do DXA, a composição corporal pode ser avaliada utilizando-se as medidas antropométricas, requerendo para tal balança, fita métrica (fita de celulose inextensível) e adipômetro

\section{Tabela 1}

Alterações metabólicas encontradas nas caquexias

\begin{tabular}{l}
\hline I. Proteicas \\
\hline Nitrogênio urinário elevado \\
Turnover proteico elevado \\
\hline Diminuição da síntese de proteínas musculares \\
Catabolismo da musculatura esquelética aumentado \\
Elevação das proteínas de fase aguda \\
\hline II. Lipídicas \\
\hline Lipólise aumentada \\
Diminuição da lipogênese \\
Hiperlipemias \\
Turnover aumentado dos ácidos graxos livres \\
Atividade da lipase lipoproteína sérica diminuída \\
Aumento da síntese de novo dos ácidos graxos \\
\hline III. Carboidratos \\
\hline
\end{tabular}

Intolerância à glicose

Hiperinsulinemia

Resistência à insulina

Aumento do turnover da glicose

Aumento da neoglicogênese

Baseado em Kotier PD. Ann Intern Méd 2000;133:622-34.

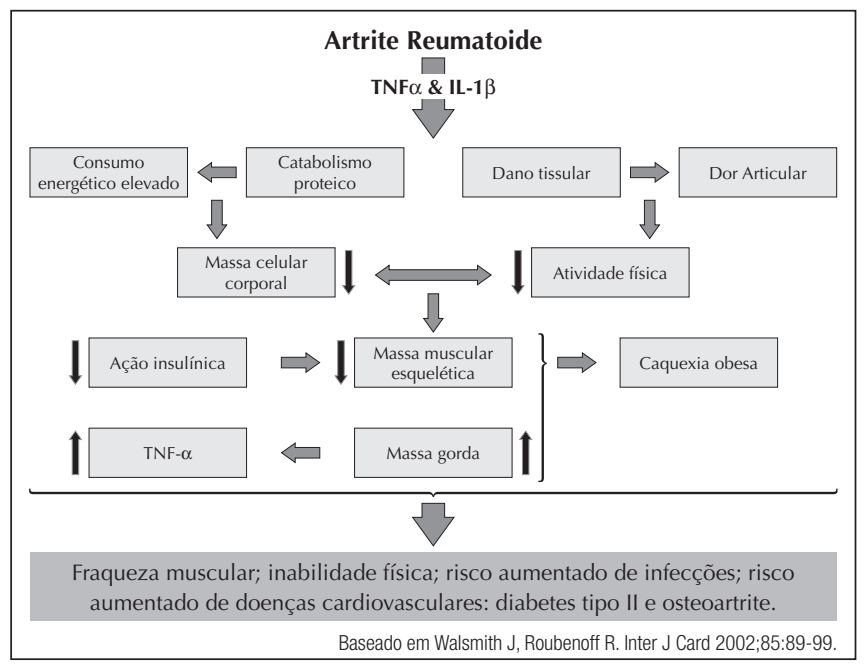

Figura1. Resumo das consequências metabólicas da artrite reumatoide. 
(compasso de Lange ${ }^{\circledR}$ ). Aplicando esses simples instrumentos, a gordura corporal pode ser indiretamente medida utilizando a relação cintura-quadril (RCQ) proposta por Ashwell. ${ }^{25} \mathrm{~A}$ realização das medidas das circunferências do braço $(\mathrm{CB})$, antebraço (CAT), abdome (CA), quadril (CQ), coxa (CC) e panturrilha $(\mathrm{CP})$ permite calcular a massa muscular (MM) e o índice de massa muscular (IMM), possibilitando o diagnóstico e classificação da sarcopenia.

O cálculo da massa muscular ( $\mathrm{MM} \mathrm{kg}$ ) é obtido pela equação de Lee. ${ }^{26}$

$M M(\mathrm{~kg})=$ estatura $^{2} \times\left(0,00744 \times\right.$ circ. braço $0^{2}+0,00088 x$ circ.coxa ${ }^{2}+0,00441 \times$ circ.pnaturrilha $)^{2}+2,4 \times$ sexo $-0,048$ $x$ idade + raça $+7,8$

Sexo $=$ homem $1 ;$ mulher 0

Raça $=-2,0$ asiáticos; 1,1 negros; 0 brancos.

O índice de massa muscular (IMM) é obtido com um cálculo simples: $\mathrm{MM}(\mathrm{kg}) /$ Estatura $(\mathrm{m})^{2}$.

É necessário salientar que na artrite reumatoide a diminuição do índice de massa muscular ocorre em decorrência do processo inflamatório, constituindo o componente sarcopênico da caquexia reumatoide, e é esse componente que podemos, através das medidas antropométricas, diagnosticar e classificar.

A classificação da sarcopenia é obtida com o índice de massa muscular (IMM) e, de acordo com a classificação proposta por Jansen, ${ }^{27-29}$ para homens considera-se normal IMM $\geq 10,75 \mathrm{~kg} / \mathrm{m}^{2}$, sarcopenia grau I $-10,75>\mathrm{IMM} \geq 8,51 \mathrm{~kg} /$ $\mathrm{m}^{2}$ e Sarcopenia grau II - IMM $<8,51 \mathrm{~kg} / \mathrm{m}^{2}$; para mulheres é normal IMM $\geq 6,75 \mathrm{~kg} / \mathrm{m}^{2}$, Sarcopenia grau I $-6,75>\mathrm{IMM} \geq$ $5,76 \mathrm{~kg} / \mathrm{m}^{2}$ e Sarcopenia grau II - IMM $<5,76 \mathrm{~kg} / \mathrm{m}^{2}$.

Mecanismos fisiopatológicos causais da caquexia reumatoide: vários fatores contribuem para o desenvolvimento da caquexia reumatoide e de seu componente sarcopênico, de modo que podemos afirmar que sua etiologia é multifatorial envolvendo insuficiente atividade física, excesso de citocinas inflamatórias, alterações hormonais, perfil energético corporal inadequado, e disfunção do turnover proteico. ${ }^{13,16,22,24} \mathrm{Na} A R$ a inflamação crônica é responsável pela alteração metabólica proteica, acelera o catabolismo e altera a relação síntesedegradação, ${ }^{22}$ facilitando, portanto, a perda de massa óssea e muscular. Dentre as citocinas ditas sarcoativas (miocitocinas) destacam-se: fator de necrose tumoral alfa (TNF $\alpha$ ou caquexina), interleucina um beta (IL-1 $\beta$ ), interleucina seis (IL-6); interferon gama (INF- $\gamma$ ) e o fator de crescimento beta (TGF- $\beta$ ). Essas citocinas, que desempenham importante papel na patogênese da $\mathrm{AR},{ }^{16}$ participam não somente do dano articular, mas também interferem na proteína corporal total e no metabolismo energético. TNF $\alpha$ é provavelmente o principal mediador da disfunção muscular. Contribui para a caquexia acelerando o catabolismo muscular e causando perda de massa muscular (componente sarcopênico). O TNF $\alpha$ atua sinergicamente com a IL-1 $\beta$, outra citocina pró-inflamatória que também contribui para a instalação da caquexia. ${ }^{22}$ Outro efeito do TNF $\alpha$ é mediar a resistência à insulina e indiretamente promover a caquexia, reduzindo a ação insulínica periférica e atenuando seu efeito anticatabólico. ${ }^{22}$ A perda de proteína muscular também é dependente da sinalização do INF- $\gamma$ e da participação do fator de transcrição nuclear kappa B (NF-kB). ${ }^{13}$

Redução no consumo alimentar pode ser outro fator contribuinte para o desenvolvimento da caquexia. A associação inversa entre a produção de IL- $1 \beta$ e a ingestão dietética sugere que a produção dessa citocina conduz à relativa anorexia, o que pode agravar a perda de massa celular corpórea característica do estado hipercatabólico de uma doença inflamatória crônica. Aumentar a ingesta, diante da alta necessidade de energia, é salutar na tentativa de melhorar o balanço energético. ${ }^{16}$

Analisando o balanço energético em uma doença inflamatória crônica, é necessário considerar o gasto energético total diário. Estudos sugerem que o gasto de energia em repouso é maior nos reumatoides; por outro lado, a energia despendida com exercícios é menor. O gasto total diário de energia (TEE total daily energy expenditure) é o somatório da energia gasta em repouso (REE - resting energy expenditure) com a energia gasta com atividade física (EEPA - expenditure of physical activity) e a energia proveniente da termogênese alimentar (TEF - thermic effect of food), de acordo com a seguinte fórmula: ${ }^{13}$ $\mathrm{TEE}=\mathrm{REE}+\mathrm{EEPA}+\mathrm{TEF}$.

Embora o REE esteja elevado na AR, ${ }^{16,30}$ a alteração do TEE depende também do EEPA. ${ }^{13}$ Baixa atividade física é responsável por $77 \%$ da diferença no TEE entre os reumatoides e os indivíduos sadios, indicando que baixa atividade física é um importante determinante da baixa TEE na AR, o que predispõe ganho de massa gorda. ${ }^{20}$.

Embora um bom controle da atividade da doença reumatoide nem sempre impeça o desenvolvimento da caquexia ${ }^{20}$ e da sarcopenia associada, certamente contribui para que o hipermetabolismo (elevado REE) seja menos acentuado. ${ }^{11}$

Consequências clínicas da caquexia reumatoide: algumas das manifestações gerais (não articulares) como sensação de fadiga, de mal estar, de indisposição, anorexia e alterações do sono ocorrem tanto nos pacientes reumatoides como nos portadores de caquexia reumatoide e devem ser atribuídas à mediação de citocinas pró-inflamatórias ${ }^{15}$ (Tabela 2). Ainda em manifestações extra-articulares há que se considerar que os músculos constituem o principal armazém de proteínas corporais, portanto a depleção desse depósito proteico (atrofia 


\section{Tabela 2}

Alterações encontradas na AR, relacionadas

às citocinas inflamatórias

\begin{tabular}{l}
\hline I. Comportamentais \\
\hline Anorexia \\
Fadiga \\
Mal-estar \\
Alterações do sono \\
\hline II. Fisiológicas \\
\hline Aumento da temperatura corporal \\
Maior gasto energético em repouso \\
\hline Diminuição da massa muscular \\
\hline "Supressão" medular \\
\hline III. Nutricionais \\
\hline Perda de peso \\
Ganho de massa gorda \\
\hline Aumento das proteínas de fase aguda \\
Balanço de nitrogênio negativo \\
\hline Hipoalbuminemia \\
Hiperinsulinemia \\
\hline Hipertrigliceridemia \\
\hline
\end{tabular}

Modificado de Kotier PD. Ann Intern Méd 2000;133:622-34.

muscular) como parte do quadro clínico da AR, contribui para o desenvolvimento do componente sarcopênico da caquexia reumatoide e intervém na capacidade do paciente de bloquear a instalação de doenças como cardiopatias isquêmicas, neoplasias ou mesmo infecções, tendo, pois, como consequência clínica maior morbidade e mortalidade. ${ }^{21}$ Além do comprometimento do aparelho locomotor, o corpo como um todo é agredido pela inflamação crônica e dois terços dos pacientes reumatoides desenvolvem caquexia. ${ }^{13}$

A caquexia da AR difere da observada em AIDS e neoplasias também em relação ao prognóstico. Nessas doenças a caquexia é prenúncio do óbito, enquanto na AR não é diretamente fatal, estaria relacionada à atividade da doença e é considerada um importante fator de comorbidade, reduzindo a expectativa de vida: $:^{22}$. A massa celular corpórea está inversamente associada ao número de juntas edemaciadas, portanto o grau de caquexia se correlaciona com a intensidade da sinovite: mais sinovite, mais caquexia. ${ }^{30}$ A caquexia aumenta a inatividade, diminuindo ainda mais a força muscular, que, por sua vez, diminui o status funcional, consolidando-se um círculo vicioso, associado à perda de independência física, facilitando a depressão e reduzindo a qualidade de vida. Como a perda de massa celular ocorre também nas vísceras e no sis- tema imunológico, consequentemente observa-se aceleração da morbidade e da mortalidade. ${ }^{22}$ Saliente-se que a perda de $40 \%$ de massa magra por si só é letal, independentemente de outros fatores de riscos. ${ }^{16,31}$

Essas considerações tornam-se particularmente importantes quando se avalia a mortalidade na população com AR. A expectativa de vida do indivíduo portador de artrite reumatoide é reduzida em duas a cinco vezes quando comparada com a população geral. ${ }^{16,32,37,38}$ Sabe-se também que, após 20 anos de doença, $80 \%$ dos pacientes apresentam algum grau de incapacidade. ${ }^{32} \mathrm{~A}$ morte por infecção nesses pacientes é 20 vezes maior do que na população geral sendo que o óbito por câncer e por doenças cardiovasculares também é mais frequente. ${ }^{34}$ De fato, estudo epidemiológico demonstrou maior risco cardiovascular em portadores de AR quando comparados a controles não reumatoides ${ }^{35} \mathrm{e}$ aqueles pacientes com índice de massa corpórea (IMC) baixo $\left(<20 \mathrm{Kg} / \mathrm{m}^{2}\right)$ têm três vezes mais risco de morte cardiovascular do que indivíduos não reumatoides com IMC normal, após correção para idade, dislipidemias, hipertensão arterial e tabagismo. ${ }^{21}$ A caquexia manifesta-se com IMC baixo e possivelmente contribui para esse maior risco cardiovascular. É importante destacar que populações não reumatoides com IMC normal e baixo não apresentam diferença no risco de morte cardiovascular, o que nos leva a concluir que o IMC é importante preditor de morte cardiovascular apenas nos pacientes portadores de AR. ${ }^{21}$

Outro importante fator a ser considerado na análise da morbidade/mortalidade na AR e sua relação com a presença de caquexia é a hiperlipemia. Nos pacientes com AR tem sido relatadas a redução da ação periférica da insulina e a diminuição da atividade física, predispondo ganho de massa gorda e hiperlipemia. ${ }^{22}$ Essas alterações metabólicas contribuem para o desenvolvimento de diabetes mellitus tipo II, osteoartrite e doença cardiovascular. ${ }^{22}$ Por outro lado, a perda de massa muscular, manifestando-se como sensação de fraqueza, contribui para a inatividade, aumentando tanto o risco cardiovascular e como o de infecções. ${ }^{16,22,36}$ As alterações metabólicas que ocorrem na caquexia reumatoide podem, por sua vez, contribuir para o risco cardiovascular aumentado, destacando-se a presença de hipertrigliceridemia (síntese de novo de triglicérides), redução da ação da lipoproteína lipase, aumento da secreção hepática de VLDL e também do pool de gordura livre. ${ }^{15}$

Considera-se que as alterações metabólicas da caquexia observadas na artrite reumatoide são mais acentuadas durante a atividade de doença. No entanto, mesmo quando a doença está bem controlada e as anormalidades metabólicas foram minimizadas, as suas consequências, como diminuição da massa magra, ganho de massa gorda e mesmo as sequelas 
funcionais, como redução da atividade física, não são corrigidas sem intervenção direta e específica. ${ }^{13}$

Tratamento da caquexia reumatoide e seu componente sarcopênico: tratar a caquexia reumatoide obrigatoriamente implica tratar a própria artrite. Em relação à caquexia, não há até o momento um tratamento padronizado. ${ }^{22} \mathrm{~A}$ Tabela 3 resume as medidas terapêuticas propostas para as caquexias. Necessário se faz focalizar inicialmente o tratamento da atividade reumatoide. Não há dúvida que a utilização de drogas antirreumáticas modificadoras do curso da doença (DMCDs) promove melhora clínica e retarda a evolução radiológica da artrite reumatoide; $;^{39,40,41,42}$ entretanto, sua eficácia sobre a caquexia e suas consequências não é tão evidente.

Da mesma forma, o uso de anti-inflamatórios não hormonais (AINEs) e corticoides (CE) visa a abortar ou a minimizar a inflamação. Os CE utilizados em altas doses, como 1000 $\mathrm{mg}$ /dia de prednisolona por três dias (pulso), são eficazes em suprimir a atividade da doença por quatro ou seis semanas. ${ }^{43}$ No entanto, apesar desse efeito anti-inflamatório e do estímulo ao apetite que produz, melhorando a ingesta alimentar, essas ações não são suficientes para minimizar os efeitos catabólicos dos corticoides, o que pioraria a caquexia. ${ }^{31}$ Em contrapartida, doses baixas, 2,5 a 7,5 mg/dia de prednisona podem proteger

\section{Tabela 3}

Medidas terapêuticas para caquexias

\begin{tabular}{l}
\hline Exercícios Físicos \\
\hline Treinamentos com pesos progressivos \\
\hline Dieta Hipercalórica \\
Estimulantes do Apetite \\
\hline Acetato de megestrol \\
\hline Medroxiprogesterona \\
\hline Dronabinol \\
\hline Agentes Anabolizantes \\
\hline Hormônio de crescimento \\
Esteroides sexuais \\
\hline Anti-inflamatórios \\
\hline Corticoides \\
\hline Indometacina \\
\hline Inibição de Citocinas \\
\hline Talidomida \\
\hline Antioxidantes \\
\hline Melatonina \\
L-Carnitina \\
\hline Ácidos graxos $\omega 3$ \\
\hline
\end{tabular}

Modificado de Kotier PD. Ann Intern Méd 2000;133:622-34. contra a perda de massa magra, por melhorar o estado funcional do paciente e por reduzir a inflamação reumatoide, mediadora do catabolismo. ${ }^{16,20,24,31}$ Sabe-se que a associação de indometacina e prednisona melhora a sobrevida de portadores de caquexia por câncer. ${ }^{45}$

A despeito do agressivo tratamento da inflamação, CE e AINEs e uso de DMCDs como metotrexato, isto não é suficiente para reverter a caquexia reumatoide, pois o hipermetabolismo pode persistir mesmo nos pacientes com bom controle clínico da atividade da doença $a^{16,18,55}$. Assim, tendo controlado a atividade da doença e conhecendo-se a fisiopatologia da caquexia, é necessário adotar intervenções focalizando a reversão do catabolismo persistente. Elas podem ser classificadas em: intervenção com exercícios, intervenção dietética e intervenção farmacológica. ${ }^{13}$

EXERCÍCIOS: os conhecimentos atuais apontam os exercícios físicos como a intervenção mais eficaz para o tratamento da sarcopenia e da caquexia reumatoide. ${ }^{48,49,50,51,52,53,54}$ A combinação de exercícios aeróbicos e treinamento com pesos progressivos é geralmente considerada a melhor intervenção para combater a caquexia, a diminuição da capacidade aeróbica, da força muscular e da resistência causadas pela artrite reumatoide. A atividade física é capaz de diminuir a sensação de fadiga, o edema articular e a rigidez matinal, bem como melhorar o desempenho físico e cardiovascular. ${ }^{22,53}$ Essas mudanças já são notadas com 12 semanas de treinamento de resistência de alta intensidade em pacientes com AR bem controlada. ${ }^{52}$ Isso sugere que essas atividades físicas, bem indicadas, considerando a presença de manifestações inflamatórias, são capazes de normalizar o metabolismo proteico na artrite reumatoide. É importante salientar que, para o treinamento de resistência ter sucesso na melhora da massa muscular, ele precisa ser acompanhado de adequada nutrição, já que o treinamento aeróbico aumenta a necessidade proteica. ${ }^{18}$

INTERVENÇÃO DIETÉTICA: as discussões sobre intervenções nutricionais na artrite reumatoide habitualmente abordam a influência de determinados elementos da dieta na artrite como um todo e não especificamente na caquexia. ${ }^{56,57,58,59}$ A suplementação com ácidos graxos poli-insaturados e monoinsaturados pode ser útil na diminuição da dor e do número de juntas edemaciadas, além de facilitar a diminuição na dose dos AINEs. A suplementação com antioxidante, como alfa tocoferol (vitamina E), proporcionaria uma importante defesa contra o aumento do estresse oxidativo. Baixas doses de ácido fólico, reposição de ferro, cálcio e vitamina D só são recomendados em situações específicas como pacientes em uso de metotrexato ${ }^{61}$, corticosteroide ou quando o diagnóstico de anemia ou de osteoporose foi clinicamente estabelecido. 
Em relação às alterações metabólicas da caquexia, pode-se dar especial atenção ao estado nutricional do paciente, prescrevendo dieta hipercalórica e insistindo na atividade física. Apesar de alguns estudos documentarem claramente os benefícios da dieta hipercalórica, há, no entanto, pacientes cuja degradação proteica é tão acelerada que a caquexia se sobrepõe ao possível ganho que a alimentação induziria. Pode-se dizer que são poucos os trabalhos publicados que avaliam as terapias nutricionais, inviabilizando uma análise crítica sobre a mensuração dos benefícios clínicos. ${ }^{13}$ Acrescentese a isso a observação de alguns autores de que a ingestão proteico-calórica dos pacientes portadores de AR é habitualmente adequada. Eles não acreditam que mesmo um consumo alimentar inadequado possa ter participação significativa no desenvolvimento da caquexia reumatoide. ${ }^{11}$ Isso posto nos parece coerente propor que as recomendações saudáveis para a população em geral, como dieta variável, bem balanceada, contendo alimentos ricos em antioxidantes, com adequada ingestão proteico-calórica, quantidades suficientes de cálcio, de ferro, vitaminas, inclusive a $\mathrm{D}$ e acrescida de poli-insaturado linolênico $(\omega 3)$ sejam recomendadas aos pacientes com AR com caquexia. Não existem dados que permitam recomendar algum tipo de dieta com requerimentos especiais.

INTERVENÇÃO FARMACOLÓGICA: alguns fármacos podem ser utilizados no combate à caquexia, a saber: estimulante de apetite, agentes anabolizantes e novos DMCDs.

Estimulantes do apetite. Experiência com HIV e doentes com câncer mostram que estimular o apetite com o acetato de megestrol melhora a ingestão alimentar e ganho de peso..$^{15}$ Considerando-se que na caquexia reumatoide o catabolismo está aumentado, é de se supor que fármacos anabolizantes como estrogênios, testosterona, nandrolona, dehidroepiandrosterona (DHEA) e hormônio de crescimento (GH) possam contribuir para a reversão da caquexia. A reposição de estrogênio em mulheres não se mostrou efetiva para aumento da massa muscular. ${ }^{9}$ Por outro lado, a reposição de testosterona em homens hipogonádicos aumenta a massa muscular. ${ }^{4,62}$ Após seis meses de terapia de reposição em homens jovens, foi possível aumentar a massa magra em 15\% dos indivíduos e diminuir a massa gorda em $11 \%$, enquanto a síntese proteica muscular aumentou $56 \%$. Porém há que se preocupar com efeitos adversos que incluem: aumento de hematócrito, aumento prostático, aumento dos níveis de antígeno prostático específico (PSA) e piora do perfil lipídico. ${ }^{4}$ Estudos com decanoato de nandrolona também mostram ganho de massa muscular, mas se fazem necessários mais trabalhos a longo prazo para avaliar o real benefício frente aos riscos.
Estudos de maior duração (nove meses) mostraram que o uso de DHEA não mostrou nenhum resultado benéfico no ganho de massa muscular ou perda de massa gorda. ${ }^{4}$ A reposição de $\mathrm{GH}$ em idosos não aumenta a força muscular nem potencializa os ganhos com treino de resistência, tampouco melhora a síntese de proteína muscular. ${ }^{4,9}$ Os principais efeitos adversos são: edema, síndrome do túnel do carpo, artralgia e ginecomastia. É importante salientar que mesmo a persistente diminuição de GH não está associada à caquexia reumatoide. ${ }^{24}$ Considerando ainda que o tratamento com GH recombinante é muito caro, cerca de US\$ 1.268 ao mês, não o recomendamos para o tratamento da caquexia.

Novos DMCDs. A resposta terapêutica aos novos DMCDs (como bloqueadores de TNF $\alpha$ ) ampliou os horizontes no tratamento da artrite reumatoide, aumentando as possibilidades de sucesso terapêutico. No entanto, mesmo o uso desses novos fármacos talvez não seja suficiente para reverter a caquexia reumatoide. ${ }^{13,15,22,44,45,46}$ Podemos especular que bloqueadores de $\mathrm{TNF} \alpha$ possam preservar a massa magra na artrite reumatoide, ${ }^{13}$ ainda que estudo recente com etanercepte por seis meses em AR inicial tenha mostrado resultado não superior ao do metotrexato. ${ }^{64}$ É possível que o bloqueio específico da principal citocina mediadora da caquexia por tempo prolongado, promova a normalização da massa magra. ${ }^{66}$ Não há muitos estudos de nosso conhecimento até a presente data examinando o efeito desses agentes na composição corporal da $\mathrm{AR}$, e futuros estudos são necessários para avaliar a real eficácia e a dose adequada para o tratamento específico da caquexia. NaTabela 4 mostram-se os possíveis tratamentos medicamentosos para caquexias.

\section{CONCLUSÃO}

A caquexia ocorre em $66 \%$ dos pacientes com artrite reumatoide e não há até o momento proposta terapêutica bem padronizada visando especificamente esse aspecto da AR. Sabemos, no entanto, que os DMCDs habituais, apesar de controlarem a atividade reumatoide e barrarem a evolução radiológica, não são suficientes para reverter a caquexia reumatoide. Modalidades terapêuticas como reposição hormonal, a exemplo de testosterona, DHEA e GH, ou têm resultados incertos ou são proibitivos frente aos efeitos colaterais. Porém, é possível que uma adequada intervenção dietética associada a corretos exercícios físicos resistidos sejam capazes de intervir com sucesso na caquexia reumatoide.

Os novos DMCDs mudaram a evolução da artrite reumatoide, controlando a atividade da doença e a progressão do dano articular. Entretanto, mais estudos são necessários para afirmar sua capacidade de reverter a caquexia. 


\section{REFERÊNCIAS}

\section{REFERENCES}

1. Mougios V. Muscle Contraction. In: Exercise Biochemistry (org.). chap 7, P 105-19, Human Kinetics, 2006.

2. Tracey KJ, Cerami A. Tumor Necrose Factor: A Pleiotropic Cytokine and Therapeutic Target. Annual Rev Med 1994;45:491-503.

3. Morley JE, Bau mgartner RN, Roubenoff R, Mayer J, Nair KS. Sarcopenia: from the Chicago Meetings. J Lab Clin Med 2001;137:231-43.

4. Greenlund LJS, Nair KS. Sarcopenia - consequences, mechanisms, and potential therapies. Mech Ageing Dev 2003;124:287-99. In www. elsevier.com/locate/mechagedeve.

5. Roubenoff R. Sarcopenic Obesity: Does Muscle Loss Cause Fat Gain? Lesson from Rheumatoid Arthritis and Osteoarthritis. Ann NY Acad Sciences 2000;904:553-7.

6. Morley JE. Anorexia of Aging: physiologic and pathologic (review). Am J Clin Nutr 1997;66:760-73.

7. Morley JE, Thomas DR. Anorexia and Aging: pathophisiology (review). Nutrition 1999;15:499-503.

8. Bau mgartner RN, Koehler KM, Romero LJ, Lindeman RD, Garry PJ. Epidemiology of sarcopenia in elderly people in New Mexico. Am J Epidemiol 1998;147:744-63.

9. Silva TAA, Junior AF, Pinheiro MM, Szejnfeld VL. Sarcopenia Associado ao Envelhecimento: Aspectos Etiológicos e Opções Terapêuticas. Rev Brás Reumatol 2006;46:391-7.

10. Morley JE, Perry HM. Androgen deficiency in aging men: role of testosterone replacement therapy. J Lab Clin Med 2000;135:370-8.

11. Poehlman ET, Copeland KC. Influence of physical activity on insulinlike growth factor-I in healthy younger and older men. J Clin Endoc Met 1990;71:1468-73.

12. Doherty TJ. Aging and Sarcopenia. J Appl Physiol 2003;95:1717-27. 
13. Rall LC, Roubenoff R. Rheumatoid cachexia: metabolic abnormalities, mechanisms and interventions. Rheumatology 2004;43:1219-23.

14. Roubenoff R, Heymsfield SB, Kehayias JJ, Cannon JG, Rosenberg IH. Standardization of nomenclature of body composition in weight loss. Am J Clin Nutr 1997;66:192-6.

15. Kotier DP. Cachexia (Review). Ann Intern Med 2000;133:622-34.

16. Roubenoff R, Roubenoff RA, Cannon JG, Kehayias JJ, Zhuang H, Dawson-Hughes B et al. Rheumatoid Cachexia: Cytokine-driven Hypermetabolism Accompanying Reduced Body Cell Mass in Chronic Inflammation. J Clin Invest 1994;93:2379-86.

17. Paget J. Nervous mimicry of organic diseases. Lancet 1873;ii:727-9.

18. Roubenoff R, Walsmith J, Lundgren N, Snydman L, Dolnikowski, Roberts S. Low physical activity reduces total. Energy expenditure in women with rheumatoid arthritis: Implications for dietary intake recommendations. Am J Clin Nutr 2002;76:774-9.

19. Roubenoff R, Roubenoff RA, Ward LM, Stevens MB. Catabolic effects of high-dose corticosteroids persist despite therapeutic benefit in rheumatoid arthritis. Am J Clin Nutr 1990;52:1113-17.

20. Roubenoff R, Roubenoff RA, Ward LM, Holland SM, Hellmann DB. Rheumatoid cachexia: depletion of lean body mass in rheumatoid arthritis: possible association with tumor necrosis factor. J Rheumatol 1992;19:1505-10.

21. Kremers HM, Nicola PJ, Crowson CS, Ballman KV, Gabriel SE. Prognostic Importance of Low Body Mass Index in Relation to Cardiovascular Mortality in Rheumatoid Arthritis. Arthritis Rheum 2004;50:3450-57.

22. Walsmith J, Roubenoff R. Cachexia in rheumatoid arthritis. Int $\mathbf{J}$ Cardiol 2002;85:89-99.

23. Arnett FC, Edworthy SM, Bloch DA, McShane DJ, Fries JF, Cooper NS et al. The American Rheumatism Association 1987 revised criteria for the classification of rheumatoid arthritis. Arthritis Rheum 1988;31:315-24.

24. Rall LC, Walsmith JM, Snydman L, Reichlin S, Veldhuis JD, Kehayias JJ et al. Cachexia in Rheumatoid Arthritis Is Not Explained by Decreased Growth Hormone Secretion. Arthritis Rheum 2002;46:2574-7.

25. Ashwell M, Chinn S, Stalley S, Garrow JS. Female fat distribution: a simple classification based on two circumference measurements. Int J Obes 1982;6:143.

26. Lee RC, Wang Z, Heo M, Ross R, Janssen I, Heymsfield SB. Total-body skeletal muscle mass: development and crossvalidation of anthropometric prediction models. Am J Clin Nutr 2000;72:796-803.

27. Janssen I, Heymsfield SB, Bau mgartner RN, Ross R. Estimation of skeletal muscle mass by bioelectrical impedance analysis. J Appl Physiol 2000;89:465-71.

28. Janssen I, Heymsfield SB, Ross R. Low relative skeletal muscle mass (sarcopenia) in older persons is associated with functional impairment and physical disability. J Am Geriat Soc 2002;50:889-96.

29. Janssen I, Bau mgartner RN, Ross R, Rosenberg IH, Roubenoff R. Skeletal muscle cutpoints associated with elevated physical disability risk in older men and women. Am J Epidemiol 2004;159:413-21.
30. Roubenoff R, Roubenoff RA, Ward LM, Holland SM, Hellman DB. Rheumatoid cachexia: depletion of lean mass in rheumatoid arthritis. Possible association with tumornecrosis factor. J Rheumal 1992;19:1505-10.

31. Roubenoff R, Roubenoff RA, Ward LM, Stevens MB. Catabolic effects of high-doses corticosteroids persistent despite therapeutic benefit in rheumatoid arthritis. Am J Clin Nutr 1990;52:1113-7.

32. Choy EHS, Panayi GS. Cytokine pathways and joint inflammation in rheumatoid arthritis. N Engl J Med 2001;344:907-16.

33. Scott DL, Symmons DP, Coulton BL, Popert AJ. Long-term outcome of treating rheumatoid arthritis: result after 20 years. Lancet 1987;1:1108-11.

34. Symmons DPM. Mortality in rheumatoid arthritis. Br J Rheumatol 1988;27:44-54.

35. Gabriel SE. The epidemiology of rheumatoid arthritis. Rjeum Dis Clin North Am 2001;27:268-81.

36. Hotamisligil GS, Murray DL, Choy LN, Spiegelman BM. Tumor necrosis fator- $\alpha$ inhibits signaling from the insulin receptor. Proc Nat Acad Sci USA 1994;91:4854-58.

37. Pincus T, Callahan LF. Taking mortality in rheumatoid arthritis seriosly: predictive markers, socioeconomic status, and comorbidity. J Rheumatol 1986;13:841-5.

38. Jacobsson LTH, Knowler WC, Pillemer S, Hanson RL, Pettitt RG, Nelson AP et al. Rheumatoid arthritis and mortality. A longitudinal study in Pima Indians. Arthritis Rheum 1993;36:1045-53.

39. Smolen JS, Sokka T, Pincus T, Breedveld FC. A proposed treatment algorithm for rheumatoid arthritis: Aggressive therapy, methotrexate, and quantitative measures. Clin Exp Rheumatol 2003;21(Supp1.31):S209-S210.

40. Smolen JS, Aletaha D, Keystone E. Superior Efficacy of Combination Therapy for Rheumatoid Arthritis. Fact or Fiction? Arthritis Rheum 2005;52:2975-83.

41. Breedveld FC, Kalden JR. Appropriate and effective management of rheumatoid arthritis. Ann Rheum Dis 2004;63:627-33.

42. Smolen JS, Steiner G. Therapeutic Strategies for Rheumatoid Arthritis. Nature Reviews Drug Discovery 2003;2:473-88.

43. Williams IA, Baylis EM, Shipley ME. Double-blind placebocontrolled trial of methylprednisolone pulse therapy in active rheumatoid disease. Lancet 1982;2:237-9.

44. Quinn MA, Conaghan PG, O'Connor PJ, Karim Z, Greenstein A, Brown A et al. Very Early Treatment With Infliximab in Addition to Methotrexate in Early, Poor-Prognosis Rheumatoid Arthritis Reduces Magnetic Resonance Imaging Evidence of Synovites and Damage, With Sustained Benefit After Infliximab Withrawal. Arthritis Rheum 2005;52:27-35.

45. Klareskog L, Heijde D, Gough A, Kalden J. de Japer JP, Malaise $\mathrm{M}$ et al. Therapeutic effect of the combination of etanercept and methotrexate compared with each treatment alone in patients with rheumatoid arthritis: double-blind randomized controlled trial. Lancet 2004;363:675-81.

46. Weiblatt ME, Keystone EC, Furst DE, Moreland LW, Weisman MH, Birbara CA et al. Adalimumab, a Fully Human Anti-tumor Necrosis Factor $\alpha$ Monoclonal Antibody, for the Treatment of Rheumatoid Arthritis in Patients Taking Concomitant Methotrexate. The ARMADA Trial. Arthritis Rheum 2003;48:35-45. 
47. Lundholm K, Gelin J, Hyltander A, Lönnroth C, Sandströn R, Svaninger G et al. Anti-inflammatory treatment may prolong survival in undernourished patients with metastatic solid tumors. Cancer Res 1994;54:5602-6.

48. Komatireddy GR, Leitch RW, Cella K, Browning G, Minor M. Efficacy of low load resistive muscle training in patients with rheumatoid arthritis functional class II and III. J Rheumatol 1997;24:1531-39.

49. Ekeblom B, Lovgren O, Alderin M, Fridstrom M, Satterstrom G. Effect of short-term physical training on patients with rheumatoid arthritis. Scand J Rheumatol 1975;4:80-6.

50. Hakkinen A, Sokka T, Kotaniemi A. Dynamic strength training in patients with early rheumatoid arthritis increases muscle strength but not bone mineral density. J Rheumatol 1999;26:1257-63.

51. Nordemar R. Physical training in rheumatoid arthritis: a controlled long-term study. Scand J Rheumatol 1981;10:25-30.

52. Rall LC, Meydani SN, Kehayias JJ, Dawson-Hughes B, Roubenoff $\mathrm{R}$. The effect of progressive resistance training in rheumatoid arthritis: increased strength without changes in energy balance or body composition. Arthritis Rheum 1996;39:415-26.

53. Rall LC, Roubenoff R, Cannon JG, Abad LW, Dinarello CA, Meydani SN. Effect of progressive resistance training on immune response in aging and chronic inflammation. Med Sci Sports Exerc 1996;28:1356-65.

54. Nordemar R, Edstrom L, Ekblom B. Changes in muscle fiber size and physical performance in patients with rheumatoid arthritis after short-term physical training. Scan J Rheumatol 1976;5:70-6.

55. Rall LC, Rosen CJ, Dolnikowski G, Hartman WJ, Ludgren N, Abad LW et al. Protein Metabolism in Rheumatoid Arthritis and Aging: Effects of Muscle Strength Training and Tumor Necrosis Factor alpha. Arthritis Rheum 1996;39:1115-24.
56. Rennie KL, Hughs J, Lang R, Jebb SA. Nutritional management of rheumatoid arthritis: a review of the evidence. J Hum Nutr Dietet 2003;16;97-109.

57. Schumacher HR, Bernhart FW, György P. Vitamin $B_{6}$ in rheumatoid arthritis: effect of treatment. Am J Clin Nutr 1975;28:1200-3.

58. Kremer JM. n-3 Fatty acid supplements in rheumatoid arthritis. Am J Clin Nutr 2000;71(suppl):349S-51S.

59. Linos A, Kaklamani VG, Kaklamani E, Koumantaki Y, Giziaki E, Papazoglou S et al. Dietary factors in relation to rheumatoid arthritis: a role for olive oil and cooked vegetable? Am J Nutr 1999;70:1077-82.

60. Dijkmans BAC. Folate supplementation and methotrexate. Br J Rheum 1995;34:1172-4.

61. Ortiz Z, Shea B, Suarez AM, Mother D, Wells G, Tugwel P. Folic and Folinic Acid for Reducing Side Effects of Patients Receiving Methotrexate for Rheumatoid Arthritis - Cochrane Review. Oxford: The Cochrane Library, 2001.

62. Gruenewald DA, Matsumoto AM. Testosterone supplementation therapy for older men: potential benefits and risks. J Am Geriatr Soc 2003;51:101-15.

63. Marcora SM, Chester KR, Mittal G, Lemmey AB, Maddison PJ. Randomized phase 2 trial of anti-tumor necrosis factor therapy for cachexia in patients with early rheumatoid arthritis. Am J Clin Nutr 2006;84:1463-72.

64. Metsios GS, Stavropoulos-Kalinoglou A, Douglas KMJ, Koutedakis Y, Nevill AM, Panoulas VF et al. Blockade of tumor necrosis factoralpha in rheumatoid arthritis: effects on components of rheumatoid cachexia. Rheumatology 2007;46:1824-7. 